\title{
Un modelo state space para la producción de energía en España
}

A model state space for the monthly Spanish energy production

\author{
Dr. Agustín ALONSO RODRÍGUEZ \\ Real Centro Universitario \\ "Escorial-María Cristina" \\ San Lorenzo del Escorial
}

Resumen: En este trabajo se estima un modelo state space para la serie de observaciones mensuales que recoge la producción y distribución de la energía total en España, desde enero de 2013 hasta enero de 2021. Tras una breve descripción de estos modelos, se pasa a su aplicación, y para destacar la valía de los mismos se comparan sus predicciones con las de otros modelos utilizados con series temporales.

Abstract: In this paper a model state space is estimated for the Monthly Spanish total production and distribution of energy, for the period January 2013 to January 2021. After a brief presentation of these models state space, the estimated model is used for prediction. To enhance the utility of these models, its predictions are compared with those generated with other models used in Time Series Analysis.

Palabras clave: Modelos state space, Filtro de Kalman, estado filtrado, estado suavizado, ARIMA, tslm, ets, paquete KFAS, paquete forecast, precisión de las predicciones.

Keywords: State space models, Kalman Filter, filtered state, smoothed state, ARIMA, tslm, ets, KFAS statistical package, forecast, statistical package, accuracy of forecasts

\section{Sumario:}

I. Introducción.

II. Modelos state space. 
III. El total de la energía en España.

IV. Estimación del modelo state space, paquete KFAS.

V. Comparaciones.

5.1. Modelo ARIMA.

5.2. Modelo de regresión.

5.3. Estimación del modelo ets, paquete forecast.

VI. Conclusiones.

VII. Bibliografía.

Recibido: septiembre 2021.

Aceptado: noviembre 2021. 


\section{INTRODUCCIÓN}

En este trabajo se estima un modelo state space para las observaciones mensuales de la serie temporal sobre la producción y distribución de la energía total en España, desde enero de 2013 hasta enero de 2021.

Para mejor evaluar la precisión de las predicciones con el modelo estimado, la serie original es particionada en dos subseries, la serie train.ts sobre la que se estima el modelo, y la serie test.ts con la que se compararán las predicciones.

Así mismo, para poner de relieve la valía del modelo state space estimado, se comparan sus predicciones con las que se obtienen con otros modelos utilizados para series temporales. En concreto, con un modelo ARIMA y con un modelo de regresión.

A pesar de ser los modelos state space un marco de referencia para los modelos de series temporales, hay que decir que hasta sólo recientemente los modelos state space no han estado a disposición de los investigadores en el ámbito del software libre, y concretamente, en el entorno del proyecto R. En este entorno, existen actualmente varios paquetes para la estimación de modelos state space. Un ejemplo notable, es el paquete $K F A S$, que es el utilizado en este trabajo.

Hay que destacar también que con un enfoque ligeramente diferente al del paquete $K F A S$, el Prof. Robert Hyndman y colaboradores tratan el modelo state space en su paquete forecast. En el último apartado de este trabajo, se estima el modelo state space con este paquete, y como se puede comprobar, la estimación suavizada o alisada del vector de estados prácticamente coincide con la del paquete $K F A S$.

\section{MODELOS STATE SPACE}

Los modelos state space constituyen un marco de referencia para un gran número de modelos utilizados con las series temporales. 
La formulación más simple de un modelo state space del tipo Gauss puede expresarse

$$
\begin{array}{r}
y_{t}=Z_{t} \alpha_{t}+\epsilon_{t} \quad \text { (ecuación de observaciones) } \\
\alpha_{t+1}=T_{t} \alpha_{t}+R_{t} \eta_{t} \quad \text { (ecuación de estados) }
\end{array}
$$

Siendo

$$
\begin{aligned}
\epsilon_{t} & \sim N\left(0, H_{t}\right) \\
\eta_{t} & \sim N\left(0, Q_{t}\right)
\end{aligned}
$$

con distribución normal, e independientes entre sí.

La ecuación (1) describe la relación entre el vector no observado de estados, $\alpha_{t}$, y el vector de observaciones $y_{t}$. La ecuación (2) describe la evolución en el tiempo del vector de estados.

El vector $y_{t}$ de dimensiones $p \times 1$ contiene las observaciones de la serie temporal, mientras que $\alpha_{t+1}$ de dimensones $m \times 1$ contien el vector de valores latentes o no observados de los estados.

Las ecuaciones (1) y (2) junto con las distribuciones de los errores, constituyen un modelo state space correctamente especificado.

Las matrices $Z_{t}, T_{t}$ y $R_{t}$ dependen del tipo de modelo, y las matrices de varianzas y covarianzas $H_{t}$ y $Q_{t}$ suelen ser invariantes en el tiempo.

El objetivo de la modelización state space consiste en lograr el conocimiento de los estados latentes $\alpha_{t}$, dadas las observaciones recogidas en $y_{t}$. El objetivo se logra, en la actualidad, gracias a los algoritmos recurrentes de Kalman: filtering y smoothing. Del primero obtenemos las predicciones un paso adelante y los errores de la predicción, es decir:

$$
\begin{array}{r}
\alpha_{t+1}=E\left(\alpha_{t+1} \mid y_{t}, \ldots, y_{1}\right) \\
v_{t}=y_{t}-Z_{t} \alpha_{t}
\end{array}
$$

así como las matrices de varianzas y covarianzas asociadas.

Sobre estos resultados, y con el algoritmo smoothing obtenemos los valores ajustados, suavizados o alisados de $\alpha_{t}$

$$
\begin{array}{r}
\widehat{\alpha_{t}}=E\left(\alpha_{t} \mid y_{n}, \ldots, y_{1}\right) \\
V_{t}=E\left(\operatorname{var}\left(\alpha_{t} \mid y_{n}, \ldots, y_{1}\right)\right.
\end{array}
$$


Con ayuda del paquete $K F A S$ en $\mathrm{R}$, del profesor Jouni Helske, de la Univeridad de Jyväskylä, Finlandia, realizamos este trabajo. Hay que señalar que KFAS sigue muy de cerca la notación de Durbin y Koopman, los autores de referencia en los modelos state space.

Llegados aquí, y a modo de paréntesis, como ya hice en mi anterior trabajo, creo interesante relacionar lo expuesto con el concepto básico de serie temporal.

Una serie temporal es una serie de observaciones ordenadas en el tiempo.

El modelo básico para representar una serie de tiempo es

$$
y_{t}=\mu_{t}+\gamma_{t}+\epsilon_{t} ; \quad t=1, \ldots, n
$$

significando los símbolos

$\mu_{t}:$ el componente tendencial, el trend

$\gamma_{t}$ : el componente periódico, el componente estacional

$\epsilon_{t}$ : el término de error o perturbación del modelo.

En economía se utiliza también la versión multiplicativa del modelo:

$$
y_{t}=\mu_{t} \gamma_{t} \epsilon_{t}
$$

$\mathrm{y}$, tomando logaritmos se pasa a la formulación aditiva en (3)

Para establecer modelos para $\mu_{t}$ y $\gamma_{t}$ se necesita el concepto de random walk, que designamos como $\alpha_{t}$ y que se expresa como

$$
\alpha_{t+1}=\alpha_{t}+\eta_{t} ; \quad \eta_{t} \sim \operatorname{iii}\left(0, \sigma_{\eta}^{2}\right)
$$

Si en (3) sustituimos $\mu_{t}$ por $\alpha_{t}$, olvidamos el componente $\gamma_{t}$, y consideramos que $\alpha_{t}$ tiene distribución normal, llegamos a

$$
\begin{array}{rlr}
y_{t} & =\alpha_{t}+\epsilon_{t} ; \quad \epsilon_{t} \sim N\left(0, \sigma_{\epsilon}^{2}\right) \\
\alpha_{t+1} & =\alpha_{t}+\eta_{t} ; \quad \eta_{t} \sim N\left(0, \sigma_{\eta}^{2}\right) \\
t & =1,2, \ldots, n
\end{array}
$$

que constituye la formulación más sencilla de un modelo state space, con $\epsilon_{t}$ y $\eta_{t}$ incorrelacionadas serialmente e independientes entre sí. La representación en (4) y (5) recibe el nombre técnico de modelo de nivel local.

En la formulación del paquete forecast antes aludido, las anteriores ecuaciones (4) y (5) pasan a escribirse como 


$$
\begin{array}{r}
y_{t}=\alpha_{t}+\epsilon_{t} \\
\alpha_{t+1}=\alpha_{t}+a \epsilon_{t}
\end{array}
$$

con un único término de error $\epsilon_{t}$. Esta formulación se denomina el modelo state space de innovación, siendo $a$ el factor de alisado o suavizado de la ecuación de estados.

\section{EL TOTAL DE LA ENERGÍA DE ESPAÑA}

En este artículo se establece un modelo state space para la serie: Production: Energy: Production and distribution of electricity, gas, steam and air conditioning: Total for Spain, datos de la OCDE, tomados del Federal Reserve Bank of Saint Louis, con fecha: 8-05-2021.

Esta serie Indice, de datos mensuales, ajustada estacionalmente y con el indice 100, en 2015 tiene un total de 553 observaciones: desde enero de 1975 hasta enero de 2021. Para facilitar las representaciones gráficas, en este trabajo se toman en consideración las observaciones desde enero del 2013, hasta enero de 2021: un total de 97 observaciones.

Por tratarse de una serie de observaciones mensuales, la serie se particiona en dos subseries, la subserie train.ts con la que se estima el modelo, y la subserie test.ts con las 12 últimas observaciones de la serie original, con cuyos valores se compararán las predicciones que se hagan

En la figura 1, podemos observar la serie completa y la subserie train.ts.

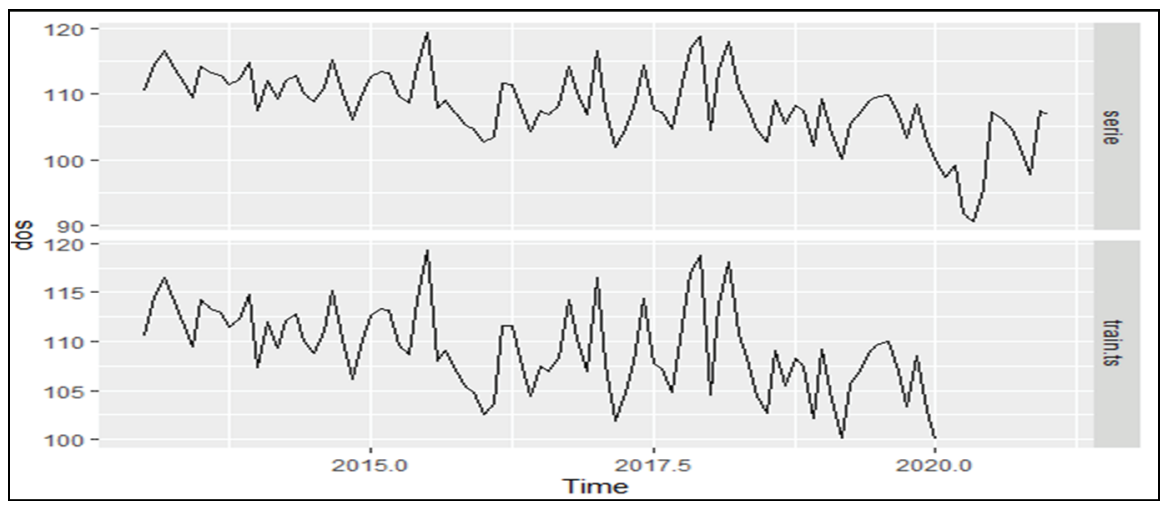

Figura 1. Serie original y s ubserie train.ts

Dado el aspecto de la serie train.ts, estimamos un modelo teniendo en cuenta el nivel como el estado de nuestro modelo. 
UN MODELO STATE SPACE PARA LA PRODUCCIÓN DE ENERGÍA EN ESPAÑA 231

\section{ESTIMACIÓN DEL MODELO STATE SPACE, PAQUETE KFAS}

Con ayuda del paquete $K F A S$ establecemos el modelo como

$$
\begin{gathered}
\text { modelo }=\operatorname{SSModel}(\operatorname{train} . \operatorname{ts} \sim \operatorname{SSMtrend}(1, Q=\operatorname{list}(\operatorname{matrix}(\mathrm{NA}))), \\
\mathrm{H}=\operatorname{matrix}(\mathrm{NA}))
\end{gathered}
$$

Los valores $N A$ representan las varianzas $\sigma_{\epsilon}^{2} \mathrm{y} \sigma_{\eta}^{2}$, que serán estimadas con la función fitSSM

Establecido el modelo, estimamos sus coeficientes mediante

$$
\text { fit }=\text { fitSSM(modelo, inits }=c(\theta, \theta) \text {, method }=\text { "BFGS") }
$$

En nuestro caso, las estimaciones de máxima verosimilitud de las varianzas son: $\sigma_{\epsilon}^{2}=0.61$ y $\sigma_{\eta}^{2}=12.71$

Aplicamos los algoritmos filtering and smoothing, que en nuestro caso podemos simplificar como

$$
\text { out }=\text { KFS (fit\$model, smoothing = "state") }
$$

En la figura 2 representamos la serie train.ts y la estimación alisada del vector de estados $\alpha_{t}$

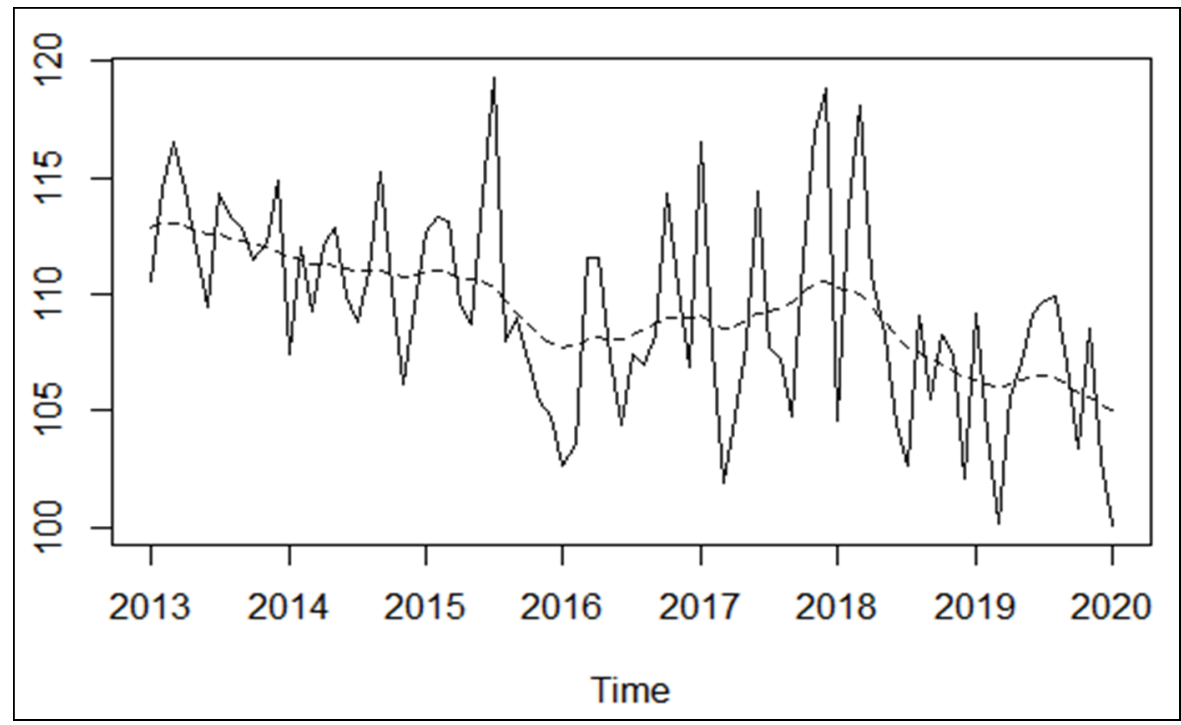

Figura 2. Series: train.ts y vector de estados suavizado 
Como se puede comprobar, el vector de estados suavizados, $\alpha_{t}$ sigue de cerca a la serie train.ts.

Como primer paso para la comprobar la validez estadística del modelo estimado, examinemos sus residuos. Dado que el algoritmo de Kalman es un algoritmo recurrente, varios son los tipos de residuos generados.

La instrucción:

$$
\text { plot(fit\$model) }
$$

permite ver los tres tipos de residuos generados. Como es una representación gráfica interactiva, que requiere el activar los distintos pasos, aquí nos limitamos a considerar los residuos recurrentes

$$
\text { rres }=\text { rstandard (out) }
$$

residuos que aparecen representados en la figura 3 , junto con la representación de la correspondiente función de autocorrelación.

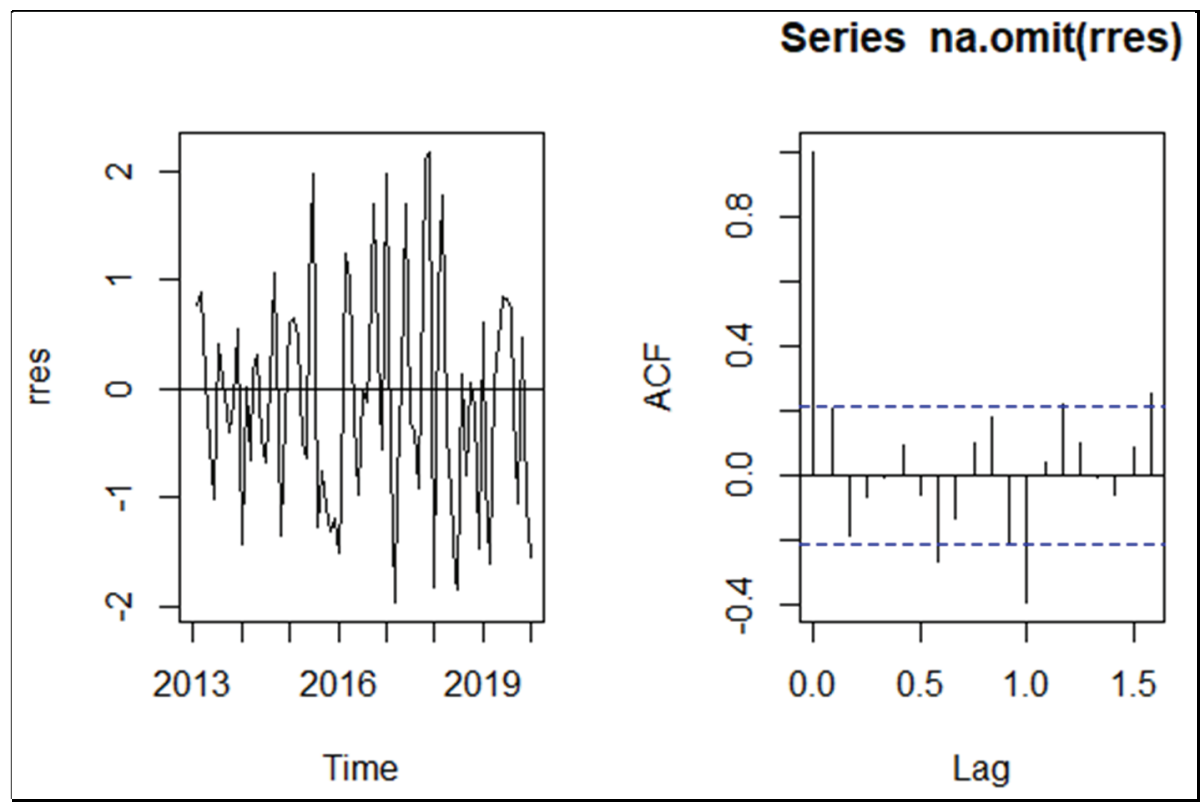

Figura 3. Residuos recurrentes y acf. de los mismos

La figura 3 muestra unos residuos que validan el modelo estimado. 
Pasemos a la fase de predicción.

Dado que en la subserie test.ts hay 12 observaciones, hagamos con nuestro modelo la predicción de 12 valores futuros. Además de las predicciones, obtenemos las bandas de confianza del $90 \%$

$$
\begin{aligned}
\text { Pred }=\text { predict (out\$model, } & \mathrm{n} \cdot \text { ahead }=12, \text { interval = "prediction", } \\
& \text { level }=0.9 \text { ) }
\end{aligned}
$$

Las predicciones aparecen en la siguiente tabla, junto con los valores de la banda de confianza. Y en la figura 4, vienen representados los valores de la serie traint.ts junto con las predicciones y la banda de confianza del $90 \%$

$\begin{array}{lrrrr}\text { \#\# } & & \text { fit } & \text { lwr } & \text { upr } \\ \text { \#\# Feb } 2020 & 105.0094 & 98.46562 & 111.5532 \\ \text { \#\# Mar } 2020 & 105.0094 & 98.34019 & 111.6786 \\ \text { \#\# Apr 2020 } & 105.0094 & 98.21708 & 111.8017 \\ \text { \#\# May 2020 } & 105.0094 & 98.09617 & 111.9226 \\ \text { \#\# Jun 2020 } & 105.0094 & 97.97733 & 112.0415 \\ \text { \#\# Jul 2020 } & 105.0094 & 97.86047 & 112.1583 \\ \text { \#\# Aug 2020 } & 105.0094 & 97.74549 & 112.2733 \\ \text { \#\# Sep 2020 } & 105.0094 & 97.63229 & 112.3865 \\ \text { \#\# Oct 2020 } & 105.0094 & 97.52082 & 112.4980 \\ \text { \#\# Nov 2020 } & 105.0094 & 97.41097 & 112.6078 \\ \text { \#\# Dec 2020 } & 105.0094 & 97.30269 & 112.7161 \\ \text { \#\# Jan 2021 } & 105.0094 & 97.19591 & 112.8229\end{array}$

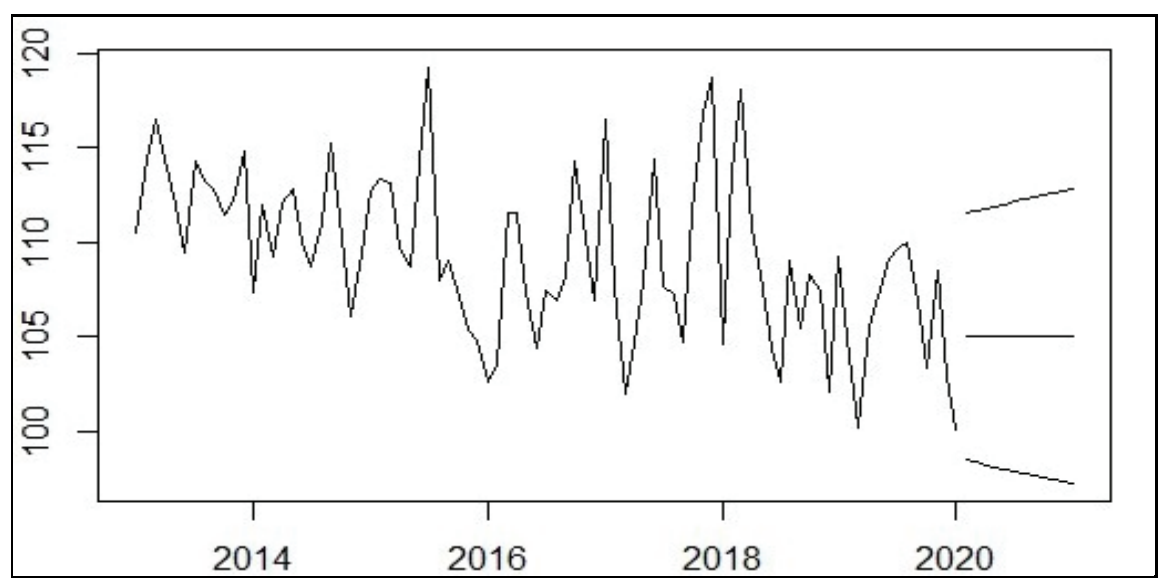

Figura 4. Subserie train.ts, y predicciones

La predicción muestra unos valores en línea recta, es decir constantes. 
Para comprobar la precisión de las predicciones, comparémoslas con los valores de la serie test.ts. Para ello acudimos a la función accuracy del paquete forecast, que genera los estadísticos

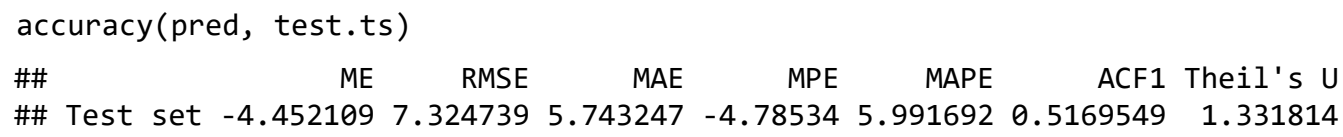

Entre estos estadísticos para establecer la precisión de las predicciones, aparece el MAPE: Mean absolute percentage error, que es uno de los más utilizados, por ser un porcentaje, independiente de la escala de medición de las observaciones y por la facilidad de su interpretación. Aunque tiene algunos inconvenientes, no obstante, se utiliza para comparar las predicciones entre distintos modelos. Hyndeman, R. y Athanasopoulos, G., en su Forecasting, Principles and Practice, segunda edición, dedican las páginas 62 a 71 para exponer el significado y las propiedades de los estadísticos arriba calculados, los más utilizados al respecto.

En nuestro caso, un error del 5.99\% podemos considerarlo como un gran ajuste de las predicciones de nuestro modelo. Es decir, la diferencia entre las predicciones de nuestro modelo y las observaciones reales es del $5.99 \%$.

\section{COMPARACIONES}

En vistas a mejor valorar el modelo state space estimado, vamos a comparar sus predicciones con las de otros dos modelos utilizados con series temporales: un modelo ARIMA, y un modelo de regresión.

\subsection{Modelo ARIMA}

El ya mencionado paquete forecast brinda la función auto.arima que de manera automática determina los valores de los distintos elementos de un modelo ARIMA, tomando como criterio de selección el estadístico de Akaike: AICc.

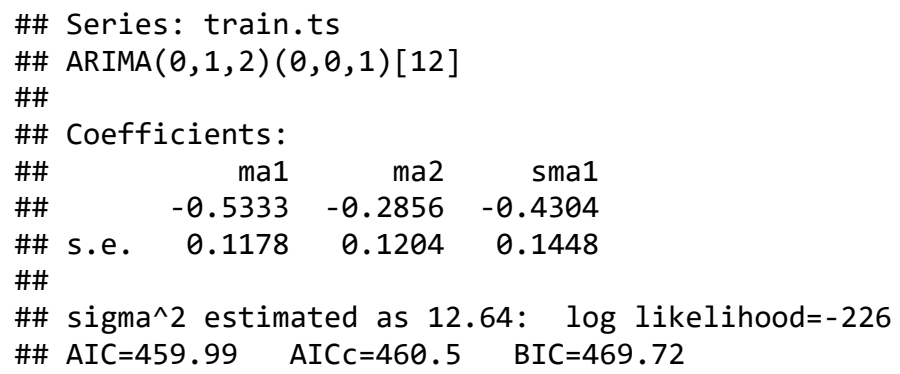


UN MODELO STATE SPACE PARA LA PRODUCCIÓN DE ENERGÍA EN ESPAÑA 235

Si pasamos a los residuos de este modelo, tenemos la figura 5.

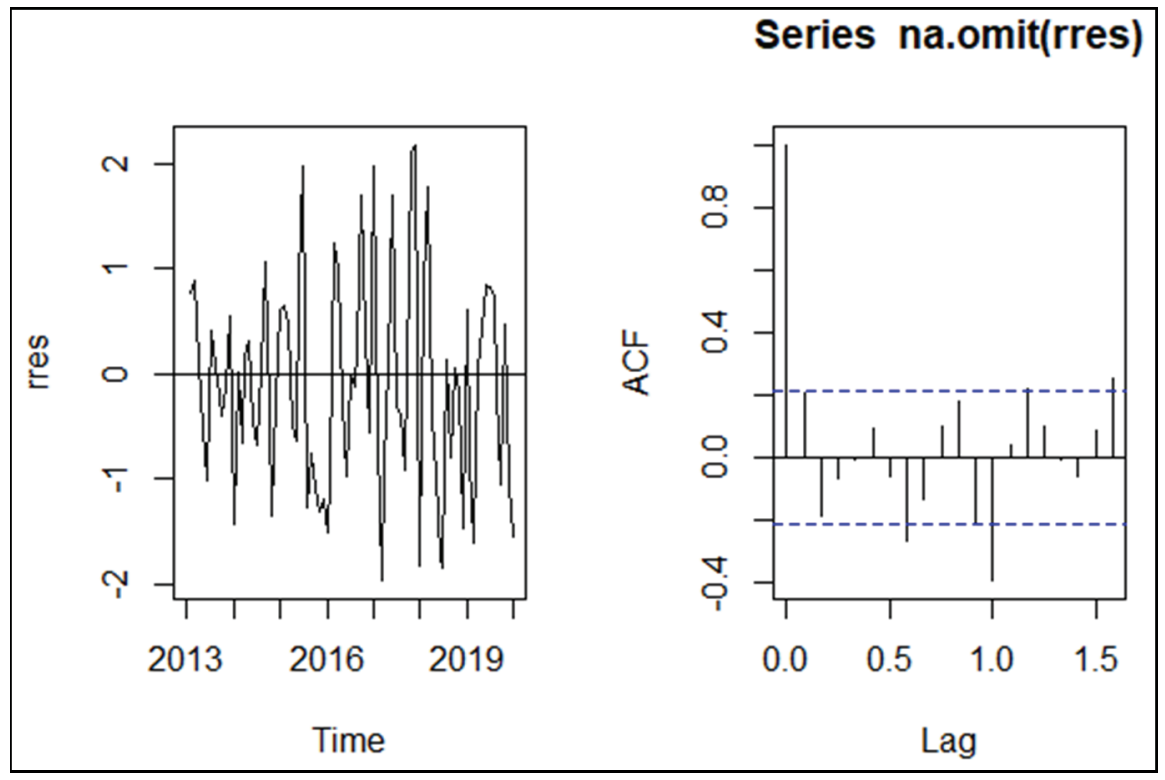

Figura 5. residuos del modelo ARIMA, y acf de los mismos

De nuevo, los residuos validan el modelo estimado.

Pasemos a las predicciones.

En la siguiente tabla aparecen los valores de las predicciones junto a las bandas del $80 \%$ y $95 \%$ de confianza. Y en la figura 6 , su representación.

$\begin{array}{llllll}\text { \#\# } & \text { Point Forecast } & \text { Lo } 80 & \text { Hi } 80 & \text { Lo } 95 & \text { Hi } 95 \\ \text { \#\# Feb 2020 } & 105.4487 & 100.89318 & 110.0043 & 98.48162 & 112.4158 \\ \text { \#\# Mar 2020 } & 107.6898 & 102.66262 & 112.7169 & 100.00140 & 115.3782 \\ \text { \#\# Apr 2020 } & 106.5395 & 101.44513 & 111.6339 & 98.74832 & 114.3307 \\ \text { \#\# May 2020 } & 106.2667 & 101.10599 & 111.4275 & 98.37406 & 114.1594 \\ \text { \#\# Jun 2020 } & 105.6272 & 100.40091 & 110.8534 & 97.63431 & 113.6200 \\ \text { \#\# Jul 2020 } & 106.0694 & 100.77841 & 111.3603 & 97.97756 & 114.1612 \\ \text { \#\# Aug 2020 } & 104.9837 & 99.62884 & 110.3386 & 96.79415 & 113.1732 \\ \text { \#\# Sep 2020 } & 107.0401 & 101.62211 & 112.4581 & 98.75399 & 115.3263 \\ \text { \#\# Oct 2020 } & 107.3864 & 101.90591 & 112.8668 & 99.00474 & 115.7680 \\ \text { \#\# Nov 2020 } & 105.0842 & 99.54203 & 110.6264 & 96.60818 & 113.5602 \\ \text { \#\# Dec 2020 } & 108.4379 & 102.83471 & 114.0412 & 99.86854 & 117.0073 \\ \text { \#\# Jan 2021 } & 109.1690 & 103.50534 & 114.8326 & 100.50720 & 117.8307\end{array}$




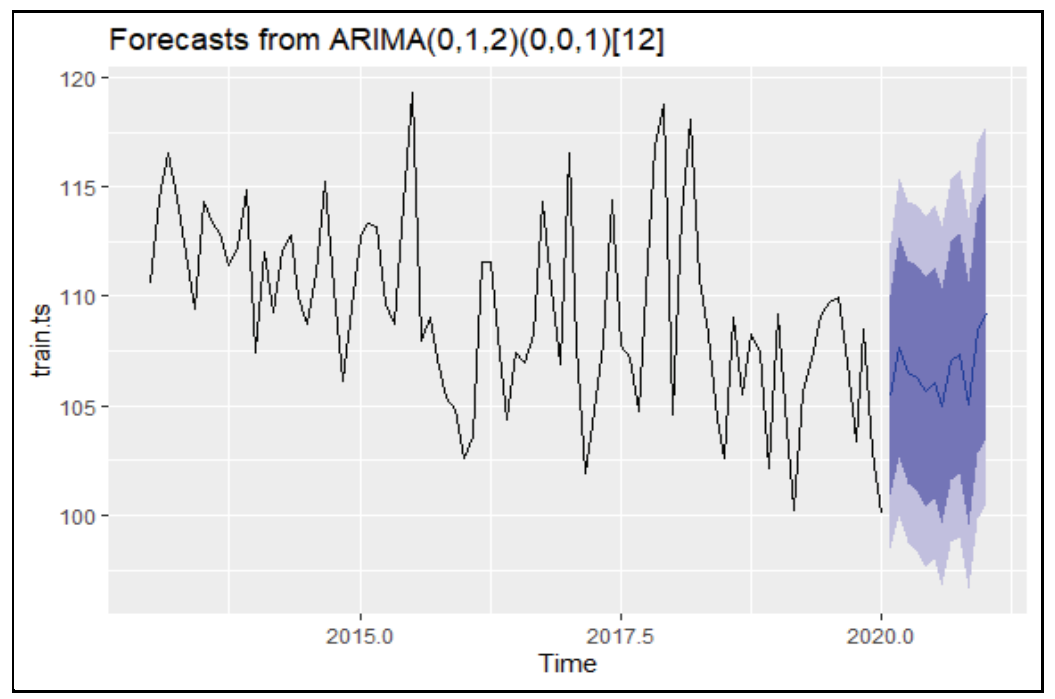

Figura 6. Serie train.ts y predicciones del modelo ARIMA

En la figura 6 aparecen la serie train.ts, las predicciones del modelo, y las bandas de confianza.

Pasemos a comparar las predicciones con las observaciones de test.ts

\begin{tabular}{llllllll} 
\#\# & \multicolumn{1}{c}{ ME } & RMSE & MAE & MPE & MAPE & ACF1 & Theil's U \\
\#\# Test set & -6.087924 & 8.177472 & 6.504054 & -6.389754 & 6.779615 & 0.6026834 & 1.488421
\end{tabular}

Acudiendo de nuevo al estadístico MAPE: 6.7796. En esta ocasión, el porcentaje de error que se comete es del 6.8\%. El modelo state space lo hace mejor.

\subsection{Modelo de regresión}

En el paquete $f p p 2$ del profesor Robert Hyndmany colaboradores se encuentra la función $t s l m$ que ajusta el modelo de regresión a series temporales.

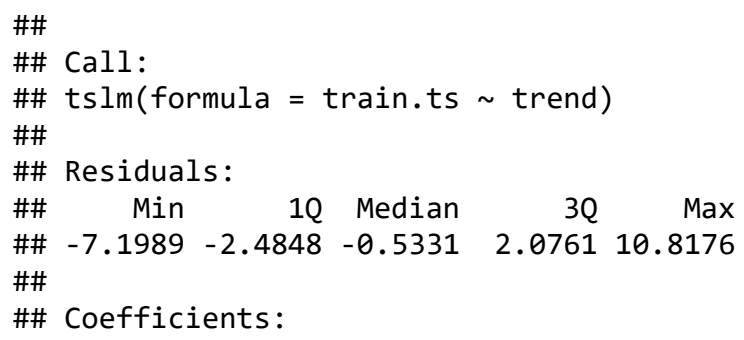




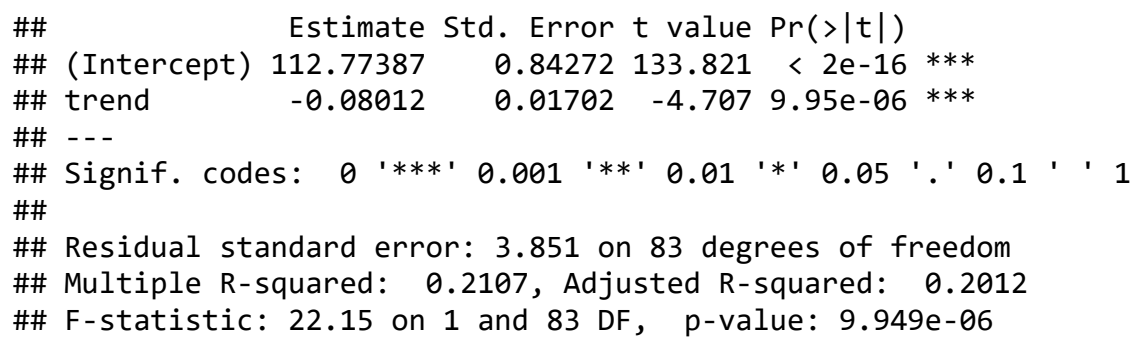

Los residuos de este modelo, aparecen en la figura 7.

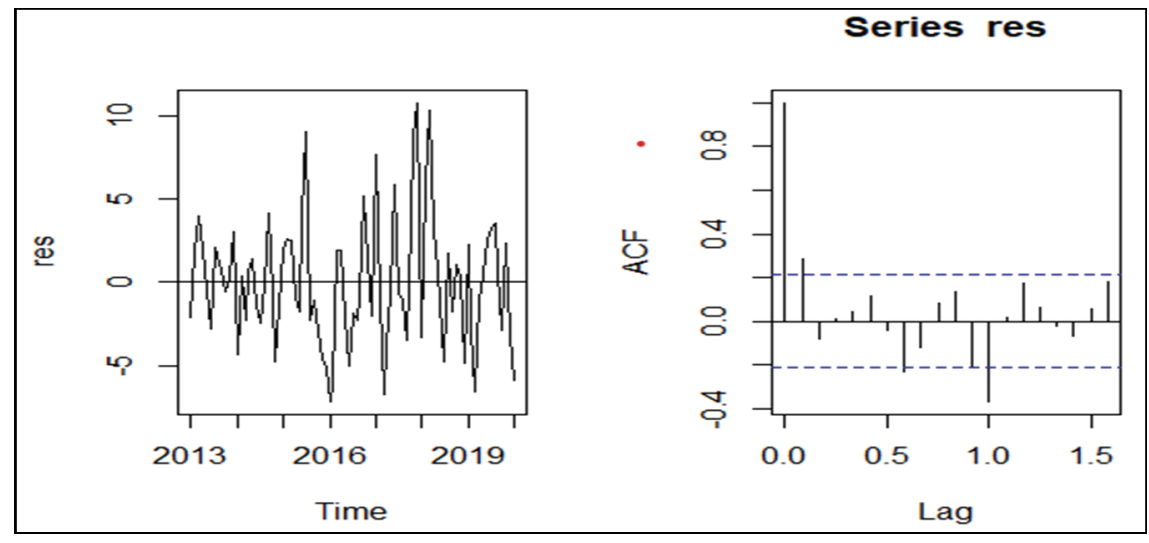

Figura 7. Residuos del modelo de regresión y acf de los mismos

Considerando que la ligera autocorrelación de los residuos no invalida el modelo estimado, pasemos a la predicción con el mismo.

En la tabla siguientes, aparecen las predicciones, y en la figura 8, su representación.

$\begin{array}{llllll}\text { \#\# } & & \text { Point Forecast Lo } 80 & \text { Hi } 80 & \text { Lo } 95 & \text { Hi } 95 \\ \text { \#\# Feb 2020 } & 105.8835 & 100.79154 & 110.9755 & 98.04370 & 113.7234 \\ \text { \#\# Mar 2020 } & 105.8034 & 100.70729 & 110.8995 & 97.95722 & 113.6496 \\ \text { \#\# Apr 2020 } & 105.7233 & 100.62295 & 110.8236 & 97.87060 & 113.5760 \\ \text { \#\# May 2020 } & 105.6432 & 100.53852 & 110.7478 & 97.78384 & 113.5025 \\ \text { \#\# Jun 2020 } & 105.5630 & 100.45399 & 110.6721 & 97.69695 & 113.4291 \\ \text { \#\# Jul 2020 } & 105.4829 & 100.36938 & 110.5965 & 97.60991 & 113.3559 \\ \text { \#\# Aug 2020 } & 105.4028 & 100.28467 & 110.5209 & 97.52273 & 113.2829 \\ \text { \#\# Sep 2020 } & 105.3227 & 100.19988 & 110.4455 & 97.43541 & 113.2100 \\ \text { \#\# Oct 2020 } & 105.2426 & 100.11500 & 110.3701 & 97.34795 & 113.1372 \\ \text { \#\# Nov 2020 } & 105.1624 & 100.03002 & 110.2949 & 97.26036 & 113.0645 \\ \text { \#\# Dec 2020 } & 105.0823 & 99.94496 & 110.2197 & 97.17263 & 112.9920 \\ \text { \#\# Jan 2021 } & 105.0022 & 99.85980 & 110.1446 & 97.08476 & 112.9197\end{array}$




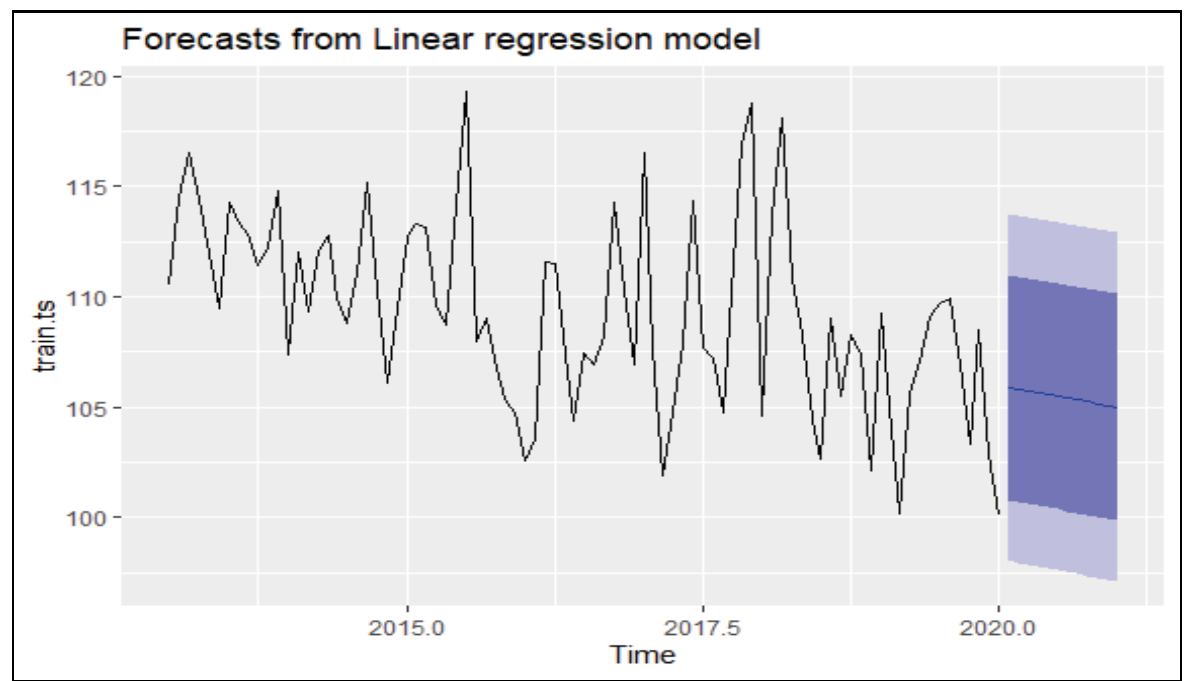

Figura 8. Subserie train.ts, y predicciones

Veamos la precisión de las predicciones.

$\begin{array}{llllllll}\text { \#\# } & \text { ME } & \text { RMSE } & \text { MAE } & \text { MPE } & \text { MAPE } & \text { ACF1 } & \text { Theil's U } \\ \text { \#\# Test set } & -4.885577 & 7.733354 & 6.02127 & -5.228066 & 6.288949 & 0.5333002 & 1.398282\end{array}$

Para este caso, el valor de MAPE es 6.2889.

\subsection{Modelo ets del paquete forecast}

La función ets permite la estimación automática de un modelo state space para series temporales, teniendo como criterio de selección la minimización del AICC.

El modelo estimado, llamémoslo fit2

$$
\text { fit2 }=\text { ets(train.ts }
$$

genera el resultado siguiente

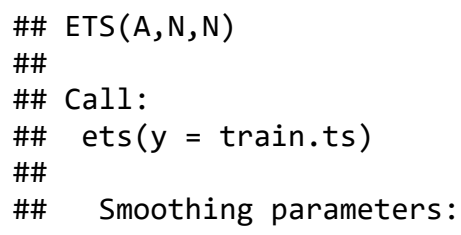




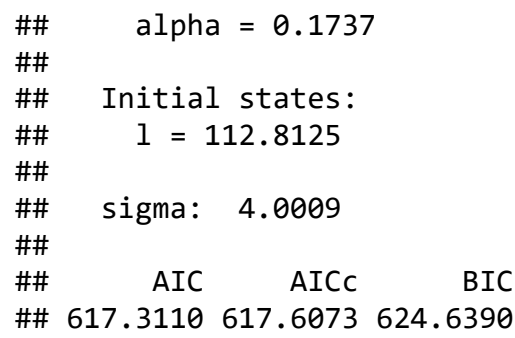

Los residuos de este modelo aparecen en la figura 9

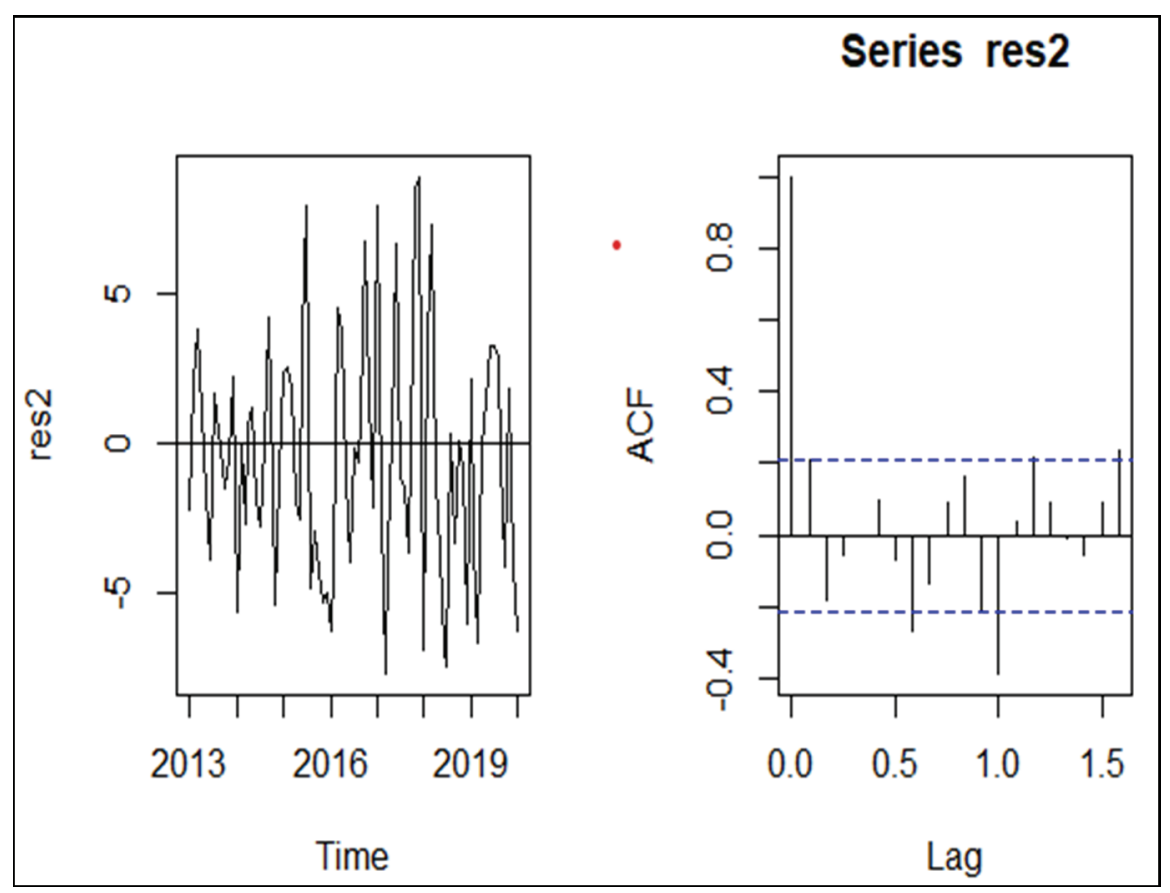

Figura 9.- Residuos del modelo ets y función acf de los mismos.

Estos residuos también validan el modelo fit 2 .

La función ets genera como resultado la serie states, que es la serie suavizada del vector de estados. Si representamos en el mismo gráfico las estimaciones suavizadas del vector de estados obtenido con el paquete $F K A S$ y con el paquete forecast, tenemos la figura 10 .

Como puede observarse, ambas estimaciones son casi coincidentes. 


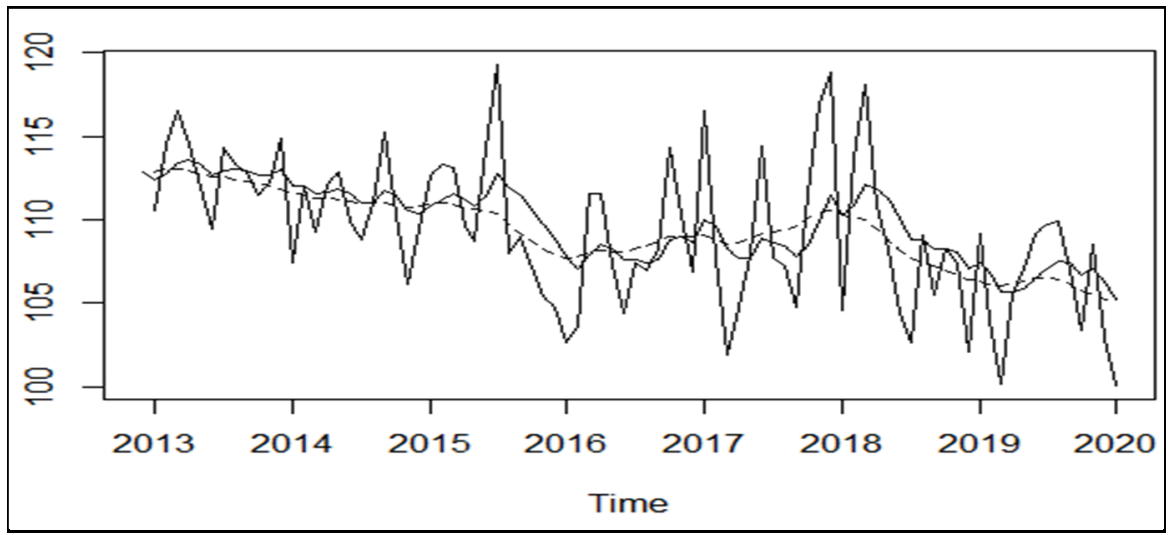

Figura 10. Serie train.ts junto a los dos estados suavizados

Pasando a la predicción, tenemos la tabla siguiente, con su representación en la figura 11

$\begin{array}{llllll}\text { \#\# } & \text { Point Forecast } & \text { Lo } 80 & \text { Hi } 80 & \text { Lo } 95 & \text { Hi } 95 \\ \text { \#\# Feb 2020 } & 105.2302 & 100.10278 & 110.3576 & 97.38850 & 113.0719 \\ \text { \#\# Mar 2020 } & 105.2302 & 100.02598 & 110.4344 & 97.27104 & 113.1893 \\ \text { \#\# Apr 2020 } & 105.2302 & 99.95029 & 110.5101 & 97.15529 & 113.3051 \\ \text { \#\# May 2020 } & 105.2302 & 99.87568 & 110.5847 & 97.04117 & 113.4192 \\ \text { \#\# Jun 2020 } & 105.2302 & 99.80208 & 110.6583 & 96.92862 & 113.5317 \\ \text { \#\# Jul 2020 } & 105.2302 & 99.72948 & 110.7309 & 96.81758 & 113.6428 \\ \text { \#\# Aug 2020 } & 105.2302 & 99.65782 & 110.8025 & 96.70799 & 113.7524 \\ \text { \#\# Sep 2020 } & 105.2302 & 99.58707 & 110.8733 & 96.59978 & 113.8606 \\ \text { \#\# Oct 2020 } & 105.2302 & 99.51719 & 110.9432 & 96.49292 & 113.9674 \\ \text { \#\# Nov 2020 } & 105.2302 & 99.44816 & 111.0122 & 96.38735 & 114.0730 \\ \text { \#\# Dec 2020 } & 105.2302 & 99.37995 & 111.0804 & 96.28302 & 114.1773 \\ \text { \#\# Jan 2021 } & 105.2302 & 99.31252 & 111.1478 & 96.17990 & 114.2805\end{array}$

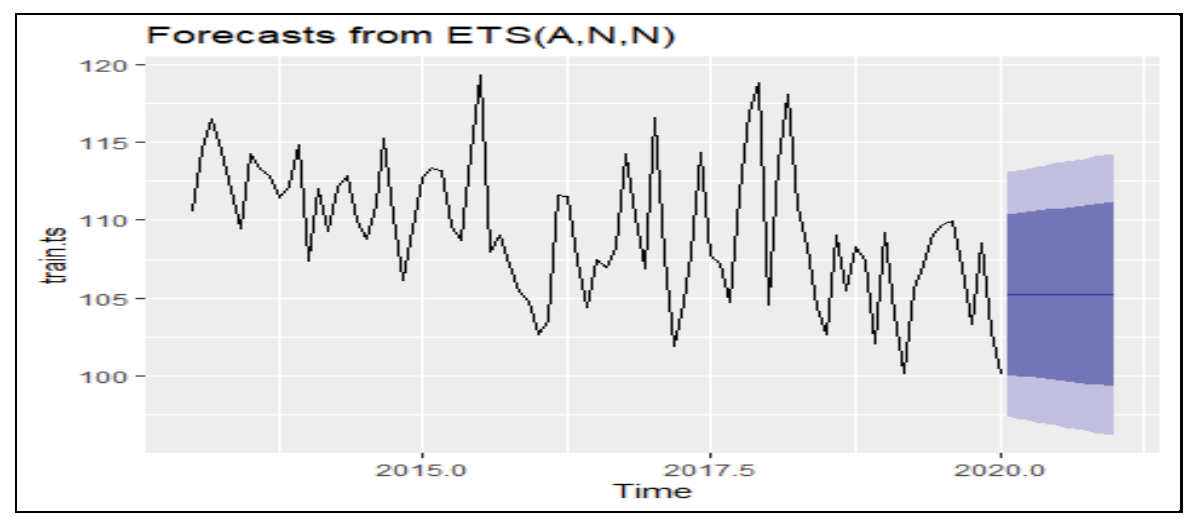

Figura 11. Serie train.ts y predicción del modelo ets. 
UN MODELO STATE SPACE PARA LA PRODUCCIÓN DE ENERGÍA EN ESPAÑA 241

Veamos la precisión de estas predicciones.

$\begin{array}{llllllll}\text { \#\# } & \text { ME } & \text { RMSE } & \text { MAE } & \text { MPE } & \text { MAPE } & \text { ACF1 } & \text { Theil's U } \\ \text { \#\# Test set } & -4.67289 & 7.460994 & 5.816841 & -5.00565 & 6.074372 & 0.5169549 & 1.355197\end{array}$

En esta ocasión, el valor del $M A P E=6.07$ es casi coincidente con el $\mathrm{MAPE}=5.99$ del modelo estimado con el paquete $F K A S$.

\section{CONCLUSIONES}

A manera de resumen podemos destacar los siguientes resultados:

En primer lugar, se ha mostrado la utilidad de los modelos state space para el análisis y predicción con series temporales;

Así mismo, se ha mostrado la versatilidad de los paquetes $K F A S$ y forecast para la estimación y predicción con estos modelos;

Por último, no obstante las semejanzas, hay que señalar la diferencia entre ambos paquetes: el paquete forecast es todo un sistema para el análisis de datos.

\section{BIBLIOGRAFÍA}

- ALONSO-RODRIGUEZ, A., La predicción de series temporales mediante el modelo de regresión, en Anuario Jurídico y Económico Escurialense (San Lorenzo del Escorial), LIV (2021) 501-520.

- ANDERSON B. D. O., y MOORE, J., Optimal Filtering, Prentice-Hall, 1979.

- DURBIN, J., y KOOPMAN, Time Series Analysis by State Space Methods, segunda edición, Oxford University Press, 2012.

- COMMANDEUR, J. J. F.; KOOPMAN, S. J., y OOMS, M., "Statistical Software for State Space Methods", in Journal of Statistical Software, 41(2011)1-18.

- COMmAndeUR, J. J. F., y KOOPMAN, S. J., An introduction to State Space Time Series Analysis, Oxford University Press, 2007.

- COWPERTWAIT, P. S. P., y METCALFE, A. V., Introductory Time series with $R$, Springer, 2009. 
- CRYER, J. D., y CHAN, K-S., Time Series Analysis with Applications in R, segunda edición, Springer, 2008.

- FRED, Federal Reserve Economic Data, https://fred.stlouisfed.org.

- HAMilTON, J. D., Time Series Analysis, Princeton University Press, 1994.

- HELSKE, J., "KFAS: Exponential Family State Space Modelsx in R", Journal of Statistical Software, 78(2017)1-39.

- HYNDMAN, R. J.; KOEHLER, A. B.; ORD, J. K., y SNYDER, R. D., Forecasting with Exponential Smoothing, The State Space Approach, SpringerVerlag Berlin Heidelberg, 2008.

- HYNDMAN, R.J., y ATHANASOPOUlOS, G., Forecasting, Principles and Practice, segunda edición, OTexts, 2018.

- HYNDMAN, R. J., y ATHANASOPOULOS, G., Forecasting, Principles and Practice, tercera edción, OTexts, 2021.

- KITAGAWA, G., Introduction to Time Series Modeling, CRC Press, 2010.

- KLEIBER, CH., y ZEILEIS, A., Applied Econometrics with R, Springer, 2008.

- OCDE, https://www.oecd.org.

- MARTIN, V.; HURN, S., y HARRIS, D., Econometric Modelling with Time Series, Specification, Estimation and Testing, Cambridge University Press, 2013.

- PETRIS, G.; PETRONE, S., y CAMPAGNOLI, P., Dynamic Linear Models with $R$, Springer, 2009.

- PELAGATTI, M. M., Time Series Modelling with Unobserved Components, CRC Press, 2016.

- R. R CORE TEAM (2021). R: A language and environment for statistical computing. R Foundation for Statistical Computing, Vienna, Austria. URL https://www.R-project.org/.

- SHUMWAY, R. H., y STOFFER, D. S., Time Series Analysis and Its Applications, with R Examples, cuarta edición, Springer, 2017.

- TUSELL, F., y "Kalman filtering in R", Journal of Statistical Software, 39(2011)1-27. 
UN MODELO STATE SPACE PARA LA PRODUCCIÓN DE ENERGÍA EN ESPAÑA 243

- WEI, W. S., Time Series Analysis, Univariate and Multivariate Methods, Addison-Wesley Publishing Company, Inc., 1990.

- WOODWARD, W. A.; GRAY, H.L., y ELLIOTT, A. C., Applied Time Series Analysis with $R$, segunda edición, CRC Press, 2017.

- ZIVOT, E., y WANG, J., Modeling Financial Time Series with S-PLUS, segunda edición, Springer, 2006. 
